\title{
Bonnes et/ou mauvaises manières médicales?
}

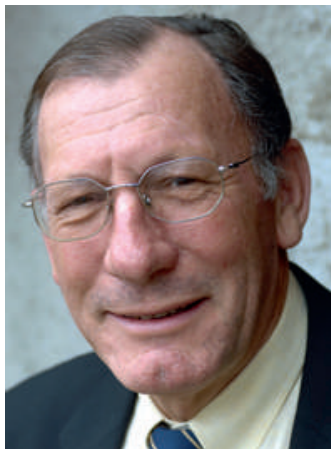

Jean Martin
Un chirurgien de notre pays a récemment posté sur le réseau "WhatsApp» une photo de lui en salle d'opération au terme d'une intervention, en blouse très tachée de sang, bras en l'air. Dans une situation à caractère professionnel donc. L'affaire a fait du bruit et l'hôpital a été interpellé.

Assumant le mandat de médecin cantonal, j'avais pour tâche, en concertation avec d'autres, d'apprécier des comportements atypiques voire critiquables de membres des professions de la santé. Il est intéressant de voir quels sont les aspects à considérer, en rapport avec les droits et les intérêts de plusieurs personnes et instances: le patient d'abord; le médecin en tant que personne; le médecin comme membre d'une profession; l'institution où il travaille (hôpital, EMS,...); éventuellement l'autorité sanitaire.

D'abord, il s'agit de s'assurer que la dignité et l'intégrité (psychique notamment) du patient ne sont pas mises en danger. Peu ou pas de problème si rien ne permet, directement ou indirectement, ni de le voir, ni de l'identifier (la situation étant différente, potentiellement sérieuse, dans le cas contraire - si par exemple, sans accord de l'intéressé, des soignants disséminent des photos où dans leur activité ils sont avec des personnalités connues). Concernant le médecin en tant que personne, qui se montre dans une position inhabituelle à un cercle large de connaissances, à lui de juger des risques qu'il prend. Noter qu'on est dans une situation différente de celle des carabins qui, en salle de garde, se livraient à des acrobaties grivoises voire choquantes - c'était à huis clos et à une autre époque. Dans le cas où messages et images sont largement distribués, sa réputation peut en souffrir; c'est son problème (ces dernières années outre-Atlantique, des carrières politiques ont été brisées pour de telles raisons). Plus avant, le médecin comme membre d'une profession/corporation, exerçant avec une autorisation officielle, peut avoir à répondre devant son association professionnelle et/ou devant l'autorité publique de surveillance. S'agissant d'écarts d'attitudes ou comportements aux conséquences négatives mineures, ils peuvent être réglés déontologiquement dans le cadre corporatif (étant relevé qu'on peut dans ce cas échapper à la sanction en démissionnant de la société professionnelle!). Dans les cas plus sérieux, des enquêtes peuvent être ouvertes par le Département cantonal de la santé. Doit aussi être traitée le cas échéant, la question des rapports avec l'employeur.

Ce sont là les principes. Au reste, dans de telles d'une certaine éducation, des «bonnes manières» [1]. La plupart d'entre nous souhaitons que les professions libérales entretiennent une image de sérieux, de correction, d'une certaine allure - même s'il n'est pas interdit de pratiquer dans une tenue vestimentaire peu avenante ou d'utiliser un langage peu châtié. Ce sera alors au/à la patient-e de tirer ses conclusions, en faisant état de son malaise, en gardant ou pas ce thérapeute.

S'agissant de «manières»: les temps ont changé et avec eux le statut du médecin. De même que les ecclésiastiques et enseignants, il n'est plus tenu de se conduire comme un très digne notable. On le voit jouer la comédie, être actif dans toutes sortes d'associations et groupes, s'engager de manière militante en politique. L'éventail des comportements qui ne font plus froncer le sourcil est devenu bien plus large. Les réseaux électroniques sont de leur côté une dimension totalement nouvelle, majeure; échanges incessants d'informations d'importance très variable. Que ce soit avec quelques dizaines ou centaines d'«amis» ou que ce soit, à l'échelon de la Toile et de la blogosphère, par des commentaires, photos ou clips visibles par des millions en quelques heures.

Il faut vivre avec son temps. Alors, le docteur ou l'infirmière qui se présente avec des tenues ou gestes «originaux», surprenants? Rien à dire? Quelque chose à dire pour le moins quand il y a une connotation discutable voire condamnable (sexuelle, violente, vulgaire, extrémiste, raciste). Là comme ailleurs, la valeur cardinale est le respect réciproque.

Les médecins et d'autres n'ont plus le devoir de paraitre en habit formel foncé, avec des airs très sérieux et en évitant tout tapage. Reste qu'il convient de se souvenir que la médecine et les soins demandent la réunion et la collaboration d'une conscience (du professionnel), de science et de compétences relationnelles et techniques, et d'une confiance, celle du malade. Cela étant, quand ces praticiens sont en vue publiquement, il parait préférable que l'observateur, et le patient tout particulièrement, ne soit pas amené à se demander s'il peut encore faire confiance à leurs qualités humaines et professionnelles.

Jean Martin, membre de la rédaction

1 Sur une thématique proche, voir: Martin J. Humour noir dans la pratique médicale, acceptable? A traiter avec discernement. BMS. 2011;92(43):1674. 